\title{
\begin{tabular}{l|l} 
Mibraries & DSpace@MIT
\end{tabular}
}

\author{
MIT Open Access Articles
}

\section{Contrast enhancement behavior of hydrogen silsesquioxane in a salty developer}

The MIT Faculty has made this article openly available. Please share how this access benefits you. Your story matters.

Citation: Nam, Sung-Wook et al. “Contrast Enhancement Behavior of Hydrogen Silsesquioxane in a Salty Developer." Journal of Vacuum Science \& Technology B: Microelectronics and Nanometer Structures 27.6 (2009): 2635. (c) 2009 American Vacuum Society

As Published: http://dx.doi.org/10.1116/1.3245991

Publisher: American Vacuum Society (AVS)

Persistent URL: http://hdl.handle.net/1721.1/74146

Version: Final published version: final published article, as it appeared in a journal, conference proceedings, or other formally published context

Terms of Use: Article is made available in accordance with the publisher's policy and may be subject to US copyright law. Please refer to the publisher's site for terms of use. 


\title{
Contrast enhancement behavior of hydrogen silsesquioxane in a salty developer
}

\author{
Sung-Wook Nam \\ Department of Materials Science and Engineering, Seoul National University, Seoul 151-742, Korea
}

Michael J. Rooks

Yale Institute for Nanoscience and Quantum Engineering, 15 Prospect St. New Haven, Connecticut 06520

Joel K. W. Yang and Karl K. Berggren

Department of Electrical Engineering and Computer Science, Massachusetts Institute of Technology, Cambridge, Massachusetts 02139

Hyun-Mi Kim, Min-Hyun Lee, and Ki-Bum Kim ${ }^{\text {a) }}$

Department of Materials Science and Engineering, Seoul National University, Seoul 151-742, Korea

Jae Hwan Sim and Do Yeung Yoon

Department of Chemistry, Seoul National University, Seoul 151-742, Korea

(Received 8 July 2009; accepted 14 September 2009; published 2 December 2009)

\begin{abstract}
The authors investigated a contrast enhancement behavior of hydrogen silsesquioxane (HSQ) in a salty development system $(\mathrm{NaOH} / \mathrm{NaCl})$. Time-resolved analysis of contrast curves and line-grating patterns were carried out to investigate the unique properties of a salty development process. In $\mathrm{NaOH}$ developer without salt, the development process was saturated beyond a certain development time. On the other hand, the addition of salt enabled a continuous development, which was not observed in the pure $\mathrm{NaOH}$ development. The continuous thinning process enhances the contrast of HSQ in the salty developer, which allows a fast collapsing behavior in HSQ line-grating patterns. During development process, salt seems to have the role of modifying HSQ by breaking network bonds preferentially, leading to a continuous development rate. (c) 2009 American Vacuum Society. [DOI: 10.1116/1.3245991]
\end{abstract}

\section{INTRODUCTION}

Hydrogen silsesquioxane (HSQ) is a widely utilized negative-type electron-beam resist material. Due to its potential for high density patterns, namely, less than $10 \mathrm{~nm}$ pitch structures, intensive investigations have been carried out to find an optimum process. ${ }^{1}$ Several development techniques, such as high temperature and high concentration hydroxide developers, have been suggested to improve the electronbeam resist performances including contrast $(\gamma)$ and sensitivity $\left(D_{0}\right)^{2,3}$ Recently, a novel development system was reported by adding salt in hydroxide developers, which offers an outstanding $\gamma$ value $(>10)$ for high resolution and high density patterning. ${ }^{4}$

Fundamental mechanisms of the cross-linking and development process of HSQ have been investigated in conventional pure hydroxide developer systems. It is known that $\equiv \mathrm{Si}-\mathrm{H}$ bonds of cagelike HSQ oligomers are converted into unstable silanols ( $\equiv \mathrm{Si}-\mathrm{OH})$ by a sequential process of $\mathrm{Si}-\mathrm{H}$ bond scission and hydrolysis reaction during electron beam or thermal activation. These silanols $(\equiv \mathrm{Si}-\mathrm{OH})$ are eventually cross-linked into network polymers $(\equiv \mathrm{Si}-\mathrm{O}-\mathrm{Si} \equiv)^{5,6}$ It was reported that developers of HSQ,usually hydroxide solutions like tetramethylammonium hydroxide (TMAH), $\mathrm{NaOH}$, and $\mathrm{KOH}$, easily dissolve cage oligomers preferentially to network polymers through the

\footnotetext{
a)Electronic mail: kibum@snu.ac.kr
}

following equation: $\equiv \mathrm{Si}-\mathrm{H} \quad$ (cage oligomers) $+\mathrm{OH}^{-} \rightarrow$ $\equiv \mathrm{Si}-\mathrm{O}^{-}+\mathrm{H}_{2} \uparrow .^{1,7,8}$ By dissolving the final product $\left(\equiv \mathrm{Si}-\mathrm{O}^{-}\right)$in developer solutions, HSQ acts as a negativetype resist material in pure hydroxide solutions. However, pure hydroxide developer solution causes the remaining HSQ to be covered with an insoluble layer, which finally stops further development and prohibits an improvement in contrast. ${ }^{9}$ The existence of this insoluble layer is critical for the design of new development systems.

In this article we studied a salty development process, $\mathrm{NaOH}(1 \mathrm{wt} \%) / \mathrm{NaCl}$ (4 wt \%), which is known to have the highest contrast $(\gamma)$ values among the results reported by Yang and Berggren. ${ }^{4}$ In terms of the contrast enhancement behavior, we investigated the role of salt in hydroxide developer through time-resolved analysis during development process. We compared both salty $(\mathrm{NaOH} / \mathrm{NaCl})$ and nonsalty $(\mathrm{NaOH})$ development processes using contrast curve variations and grating-line patterns. The modification of chemical bonding of HSQ by the addition of salt was analyzed with Fourier transform infrared (FTIR). The pure $\mathrm{NaOH}$ saturates the development process whereas the salty continues to develop as the development time increases. This continuous development is a key property of salty developer, leading to contrast enhancement.

\section{EXPERIMENT}

HSQ solution (XR1541, 6\%) from Dow Corning Co. was spun on silicon nitride coated silicon substrates to a thickness 

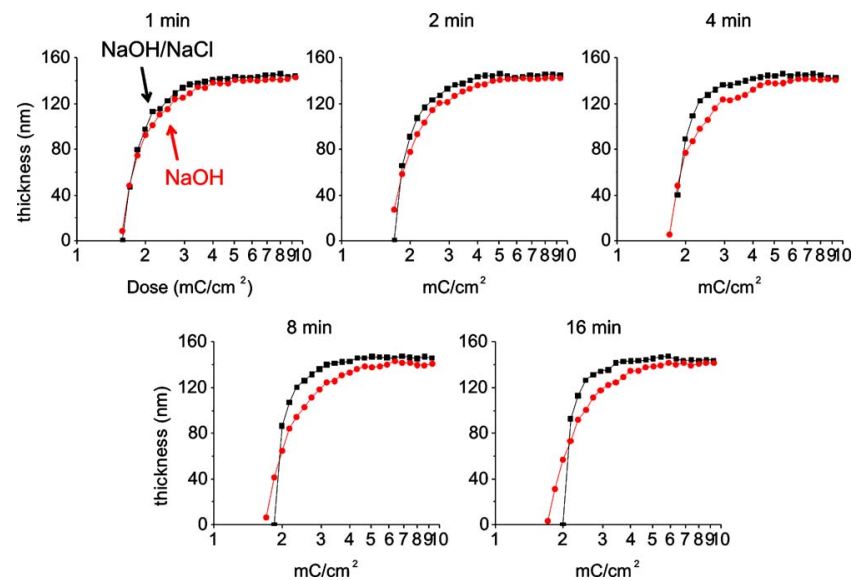

Fig. 1. (Color online) Time-resolved contrast curves (1-16 $\mathrm{min})$ for both the salty (squares) $(\mathrm{NaOH} / \mathrm{NaCl})$ and the nonsalty (circles) $(\mathrm{NaOH})$ developers.

of $\sim 140 \mathrm{~nm}$. Prebake was not performed since baking process degrades the $\gamma$ and the pattern resolution. Electron-beam exposures were carried out on a Vistec VB-6 electron-beam system operating at $100 \mathrm{keV}$. For contrast curve characterization, $\sim 20 \times 50 \mu \mathrm{m}^{2}$ wedge patterns were exposed with an electron-beam current of $\sim 100 \mathrm{nA}$. The area doses were controlled from 1 to $10 \mathrm{mC} / \mathrm{cm}^{2}$. In the case of grating-line patterns (1 mm long), we used $40 \mathrm{nA}$ electron-beam current. The exposed patterns were developed by a salty solution $(\mathrm{NaOH} 1 \mathrm{wt} \% / \mathrm{NaCl} 4 \mathrm{wt} \%)$ and a nonsalty solution $(\mathrm{NaOH} 1 \mathrm{wt} \%)$ for various times from 1 to $16 \mathrm{~min}$ at room temperature. The thickness of the remaining HSQ was measured with a surface profilometer. To see the cross-sectional view of grating lines, we used a JEOL JSM-7401F scanning electron microscope (SEM). For analysis of chemical bonding status of HSQ, FTIR measurement was performed.

For the investigation of aging effects of HSQ, we used HSQ solution (XR1541, 4\%) and $200 \mathrm{keV}$ AIPEL system which is a projection-type electron-beam lithography tool modified from transmission electron microscope. ${ }^{10}$ The remaining resist thickness was measured with an opticalmeasurement system (Nanospec AFT/4150, Nanometrics).

\section{RESULTS AND DUSCUSSION}

Figure 1 shows the time-resolved contrast curves in both salty and nonsalty developers. The difference in curve shape between the two developers becomes more distinct for longer development times. In the case of nonsalty developer, there are no noticeable changes as the development times are varied. However, the contrast curves of salty developer continue to change as the development time increases. In order to evaluate the development process, we plotted the values of $\gamma$ and $D_{0}$ as a function of development time in Figs. 2(a) and 2(b), respectively. In the case of nonsalty developer, the $\gamma$ decreases slightly and saturates to $\sim 4$ after $4 \mathrm{~min}$. On the other hand, the salty developer allows $\gamma$ to increases continuously up to $\sim 12$. The $D_{0}$ plot shows that the nonsalty devel- (a) Contrast

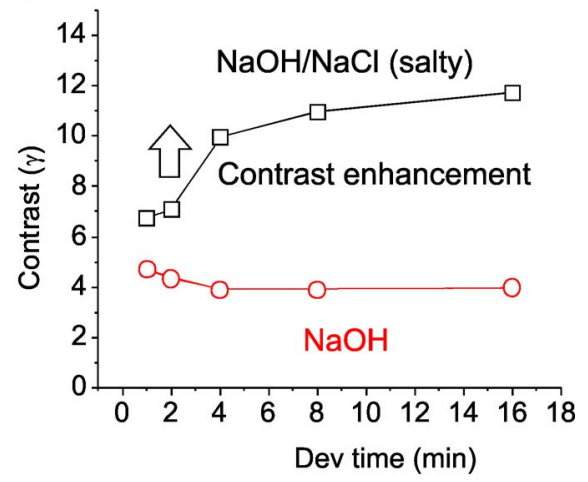

(b) Sensitivity

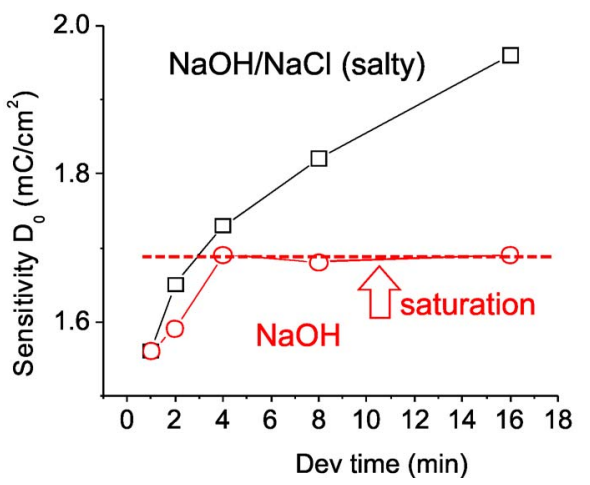

FIG. 2. (Color online) Variations in (a) $\gamma$ and (b) $D_{0}$ as the function of development time. The salty developer increases $\gamma$ up to $\sim 12$, whereas the $\gamma$ of the nonsalty developer saturates after $4 \mathrm{~min}$.

oper causes the development process to stop after $4 \mathrm{~min}$, whereas the salty developer allows development to continue.

For a more precise analysis of the development process, we plot the remaining HSQ thickness during development for different electron-beam doses, which provides an alternative view of time-resolved contrast curves (Fig. 3). Interestingly, at the early stages of the development process, both salty and nonsalty developers show similar behaviors, such as a sharp decrease in thickness followed by a gradual saturation. However, as the development time is further increased, the salty developer continues to thin the resist film, whereas the nonsalty developer does not lead to any significant changes in resist thickness. This continuous thinning is unique to the salty development system.

In the salty development system, the gradual saturation of the remaining thickness followed by the continued thinning behavior enhances the $\gamma$ significantly, which makes it different from other developers. For instance, conventional hydroxide developer such as TMAH is known to saturate and stop the development, which is attributed to an insoluble layer formation on the HSQ surface. ${ }^{9}$ This saturation prevents further $\gamma$ improvement once $\gamma$ is determined by the initial thinning at the very early stages of development. On the other hand, the salty developer for HSQ provides a continuous thinning process, leading to a time-dependent $\gamma$ variation. This continuous thinning process is typically observed in the medium-range dose such as $2-2.16 \mathrm{mC} / \mathrm{cm}^{2}$ of 
(a) $\mathrm{NaOH} / \mathrm{NaCl}$ (salty)

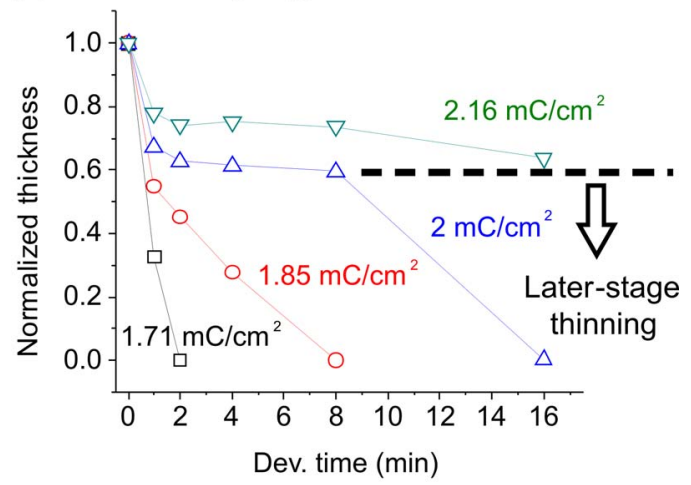

(b) $\mathrm{NaOH}$

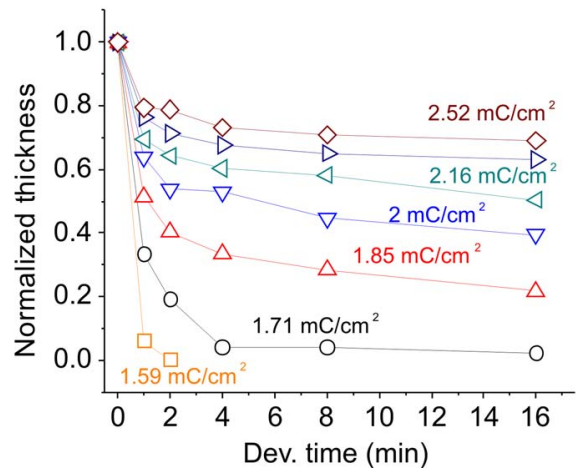

FIG. 3. (Color online) HSQ thickness variations vs development time for each electron-beam dose for (a) the salty and (b) the nonsalty developers. Typically, in the case of $2 \mathrm{mC} / \mathrm{cm}^{2}$, the salty process leads to the later-stage thinning. However, the nonsalty process does not exhibit any noticeable further thinning.

the salty developer, whereas the HSQ at the high-range dose is still saturated, as shown in Fig. 3(a). The later-stage thinning is the critical process which enhances contrast.

In order to illustrate the efficacy of the salty developer for patterned structures, we carried out time-resolved analysis for grating-line patterns (Fig. 4). We used three different line patterns which have different line doses, such as 46,33 , and $23.6 \mathrm{nC} / \mathrm{cm}$. We developed them for different times from 1 to $16 \mathrm{~min}$ in both nonsalty and salty developers and characterized the evolution of the surface profile with SEM cross-sectional images.

Figure 4(a) shows the high-range dose grating lines $(46 \mathrm{nC} / \mathrm{cm})$ which are partially merged, as can be seen in the cross-sectional view. As the development time is increased, the nonsalty developer does not induce significant changes in surface profile, whereas the salty developer starts to define the line structures after some period (4-8 min). Figure 4(b) shows that the medium-range dose patterns $(33 \mathrm{nC} / \mathrm{cm})$ obviously have the definition of separate lines, but the evolutions of resist profiles are quite different for salty and nonsalty solutions. The nonsalty developer tends to develop patterns isotropically, which is similar to an isotropic wet etching process. This process cannot improve the resolution significantly since the height of patterns is also decreased as well as the linewidth [inset of Fig. 4(b)]. On the other hand, the salty developer decreases the linewidth much faster than (a) $46 \mathrm{nC} / \mathrm{cm}$ (high-range dose)

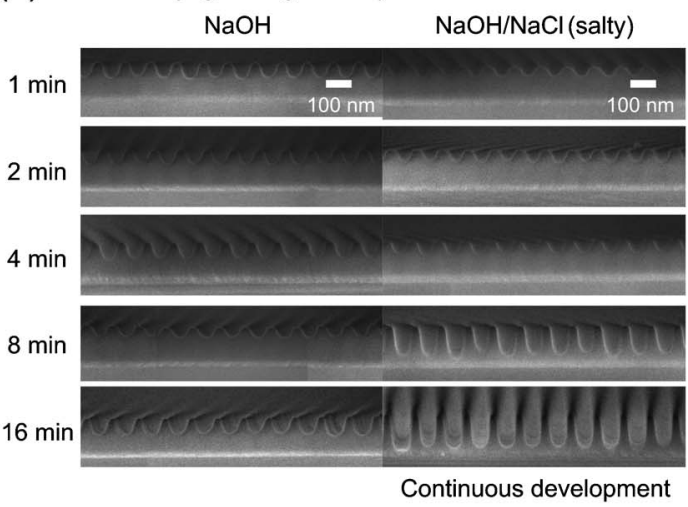

(b) $33 \mathrm{nC} / \mathrm{cm}$ (medium-range dose)

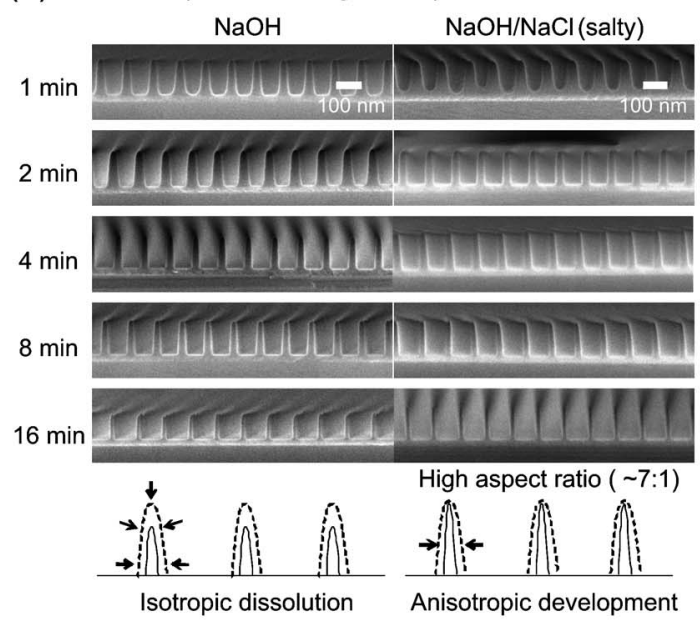

(c) $23.6 \mathrm{nC} / \mathrm{cm}$ (low-range dose)

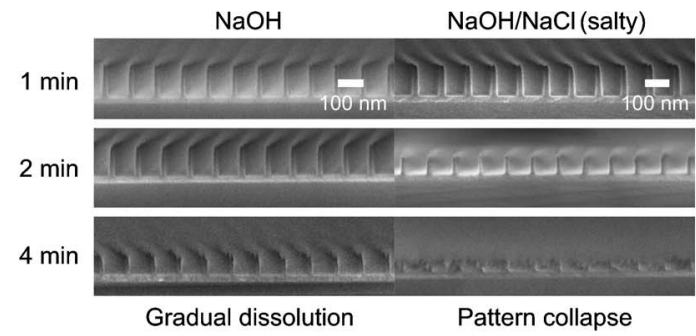

FIG. 4. Time-resolved cross-sectional SEM analysis of HSQ line-grating patterns for the salty and the nonsalty developer for different electron-beam line doses (a) $46 \mathrm{nC} / \mathrm{cm}$ (high-range dose), (b) $33 \mathrm{nC} / \mathrm{cm}$ (medium-range dose), and (c) $23.6 \mathrm{nC} / \mathrm{cm}$ (low-range dose). HSQ grating-line patterns are prepared on $\mathrm{Si}_{3} \mathrm{~N}_{4}$-deposited $\mathrm{Si}$ substrate to protect $\mathrm{Si}$ from $\mathrm{NaOH}$ wet etching.

decreasing the film thickness [inset of Fig. 4(b)]. This anisotropic development comes from the high $\gamma$ behavior of the salty development, which enhances patterning resolution. Figure 4(c) shows the low-range dose patterns $(23.6 \mathrm{nC} / \mathrm{cm})$ in which the nonsalty developer induces the gradual dissolution of line patterns, whereas the salty developer leads to even faster dissolution once the patterns start to collapse. This faster collapsing behavior of the salty developer is most likely due to the continuous thinning process.

To investigate the role of the salt in $\gamma$ enhancement behavior, we assumed that the addition of salt modifies the 


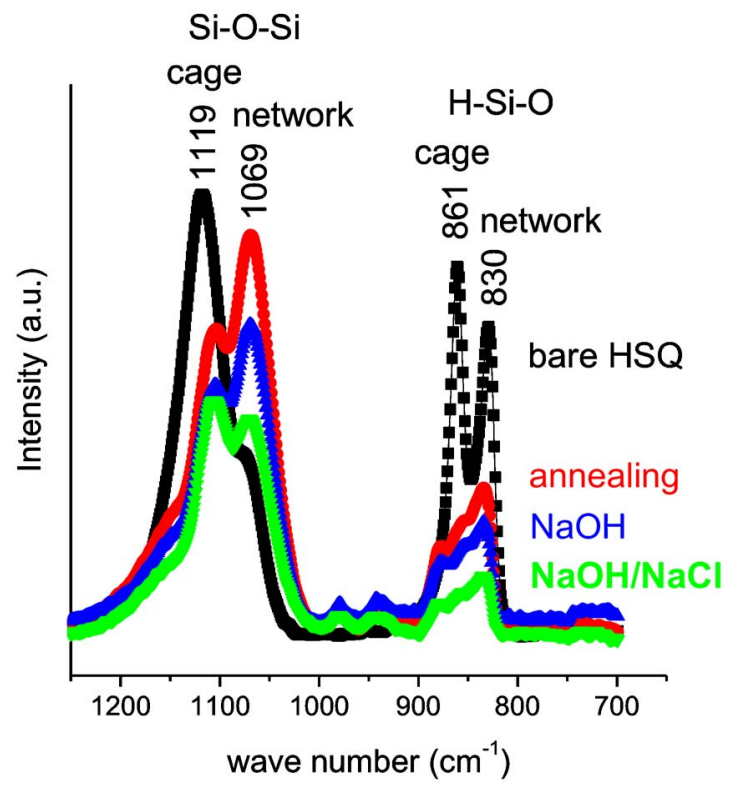

FIG. 5. (Color online) FTIR analysis for annealed HSQ films for salty and nonsalty developers. All HSQ started with $140 \mathrm{~nm}$ thickness (bare HSQ) during spin coating. Annealing process was carried out at $400{ }^{\circ} \mathrm{C}$ for $1 \mathrm{~h}$ in vacuum, which shrank the thickness to $110 \mathrm{~nm}$. Annealed HSQ samples were dipped in both salty and nonsalty developers for $45 \mathrm{~s}$. The final thickness of developed HSQ was decreased to $80 \mathrm{~nm}(\mathrm{NaOH})$ and $70 \mathrm{~nm}$ $(\mathrm{NaOH} / \mathrm{NaCl})$. The FTIR peak heights were not normalized, so the relative difference in peak heights may reflect the difference in thickness and density of HSQ films. In the salty developer, the network peak in $\mathrm{Si}-\mathrm{O}-\mathrm{Si}$ stretch is smaller than the remaining cage-bond peak.

chemical state of HSQ during development. Usually, hydroxide developers such as TMAH or $\mathrm{NaOH}$ are likely to form an insoluble layer on the HSQ surface, which stops the development process. ${ }^{9}$ The insoluble layer is known to consist of siloxane-based network bonds, as confirmed by x-ray photoelectron spectroscopy and FTIR spectroscopy. ${ }^{3,9}$ In order to analyze the change in chemical status during development, we carried out FTIR analysis by using annealed HSQ films. ${ }^{11}$ The annealed HSQ film $\left(400{ }^{\circ} \mathrm{C}\right.$ for $\left.1 \mathrm{~h}\right)$ has noticeable network-bond peaks $\left(1069\right.$ and $\left.830 \mathrm{~cm}^{-1}\right)$ in $\mathrm{Si}-\mathrm{O}-\mathrm{Si}$ stretch, which clearly shows the polymerization process during annealing (Fig. 5). This annealing was supposed to correspond to the medium-range dose based on our estimations about the development rate according to the various annealing conditions. To estimate the effect of developers, we dipped the annealed HSQ films in both nonsalty and salty developers. The FTIR analysis shows that the network-bond peak $\left(1069 \mathrm{~cm}^{-1}\right)$ of salty development was less than that of the undeveloped film. Considering that the FTIR signal comes from the HSQ volume, the significant decrease in the network-bond peak reflects that the role of the salt is not limited in the surface but effective to the whole HSQ. However, we believe that the surface of HSQ is more dominantly affected by the developers, thus the average FTIR signal shows the low network-bond peak. An addition of salt appears to break network bonds preferentially, which may increase the development rate and enhance the contrast.

\section{(a) Aged sample}

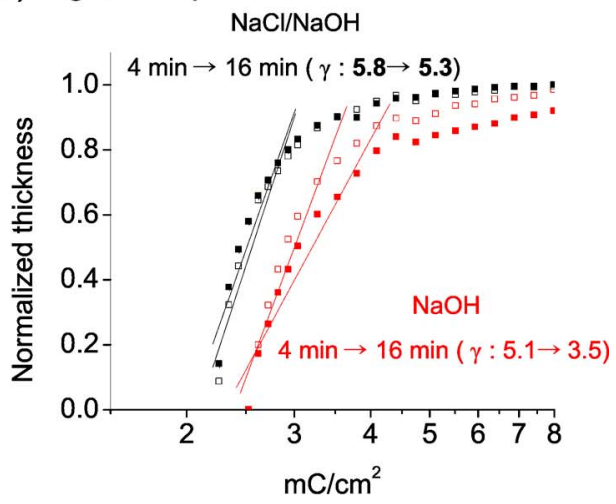

(b) Immediately-processed sample

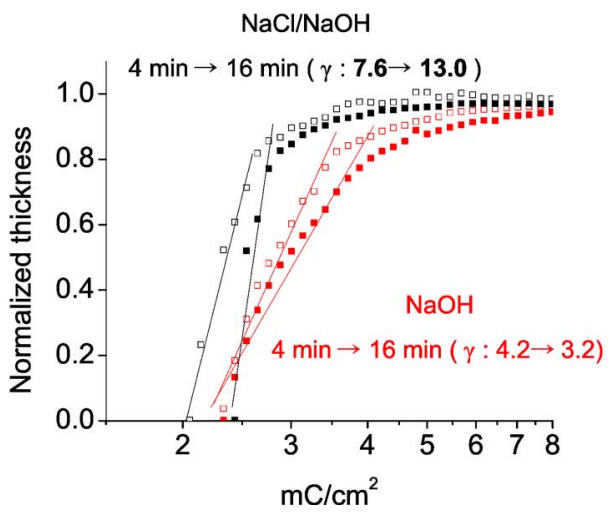

FIG. 6. (Color online) Aging effect of HSQ on the salty development system for two different samples; (a) aged HSQ and (b) HSQ in which all processes were done within a day. Only the salty development process is sensitive to an aging effect, whereas the nonsalty development is not affected by aging.

The importance of chemical bond state in the salty development system can be supported by studying aging effects (Fig. 6). We prepared two samples: one is an aged sample which was developed more than 2 weeks after the e-beam exposure has been done and the other a sample for which all process (spin coating, electron-beam exposure, development, and thickness evaluation) was done within a day. In the case of the aged sample, " 2 weeks" was arbitrarily chosen, and the sample was stored in air. Contrast curve variations for two development times (4 and $16 \mathrm{~min}$ ) are shown in Fig. 6. The curves clearly show an aging effect when using the salty developer. In the case of the nonsalty developer, there was little difference between the aged sample and the immediately processed sample. As the development time becomes longer, the development process stops for both samples. This tendency coincides with our experience that there were no significant aging effects in conventional hydroxide development (such as TMAH) even if we developed HSQ a month after spin coating. This means that the nonsalty development process is much less sensitive to resist aging. It is likely that the cessation of development when using hydroxides is related to the insensitivity to aging. ${ }^{9}$ In contrast, the salty development process was very sensitive to aging. Only by developing immediately were we able to detect a continuous development process with noticeable $\gamma$ enhancement behav- 
ior $(\gamma=7.6$ at $4 \mathrm{~min} \rightarrow \gamma=13$ at $16 \mathrm{~min})$. The aged sample had low $\gamma(5-6)$ and did not show any $\gamma$ enhancement as the development time was extended. This sensitivity to aging might be attributed to the gradual cross-linking of HSQ in an ambient atmosphere. Cross-linking over time might inhibit the function of network-bond breaking of the salty developer. Since the nonsalty developer does not have any function of network-bond breaking, the nonsalty process seems to be less affected by the cross-linking of an aging.

\section{CONCLUSION}

We have investigated contrast enhancement behavior of HSQ in a salty development system through time-resolved analysis during development. An addition of the salt in a hydroxide solution enabled a continuous development process, unlike that of conventional pure hydroxide developers. The continuous thinning behavior of the salty developer is critical to contrast enhancement. Thinning over long development times was demonstrated using the analysis of grating-line patterns. The FTIR analysis shows that the addition of salt modifies the chemical status of HSQ during the development process by breaking network bonds preferentially. This chemical modification allows the resist to thin continuously and enhances the $\gamma$ of HSQ.

\section{ACKNOWLEDGMENTS}

The authors thank IBM Research (Yorktown Heights, $\mathrm{NY}$ ) for the use of their electron-beam lithography facility.
The work at Seoul National University was supported by the Frontier Research Program of Tera-Level Nanodevices (TND) funded by the Korean Ministry of Science and Technology (MOST). One of the authors (S.-W.N.) acknowledges financial support by the Brain Korea (BK)-21 program funded by the Ministry of Education in Korea. The work at MIT was supported by the NRI/INDEX program and the King Abdulaziz City for Science and Technology and Alfaisal University.

${ }^{1}$ J. K. W. Yang, B. Cord, K. K. Berggren, J. Klingfus, S. W. Nam, K. B. Kim, and M. J. Rooks, J. Vac. Sci. Technol. B 27 (2009) (in press).

${ }^{2}$ W. Henschel, Y. M. Georgiev, and H. Kurz, J. Vac. Sci. Technol. B 21, 2018 (2005).

${ }^{3}$ M. Haffner, A. Haug, A. Heeren, M. Fleischer, H. Peisert, T. Chasse, and D. P. Kern, J. Vac. Sci. Technol. B 25, 2045 (2007).

${ }^{4}$ J. K. W. Yang and K. K. Berggren, J. Vac. Sci. Technol. B 25, 2025 (2007).

${ }^{5}$ M. J. Loboda, C. M. Grove, and R. F. Schneider, J. Electrochem. Soc. 145, 2861 (1998).

${ }^{6}$ H. Namatsu, Y. Takahashi, K. Yamazaki, T. Yamaguchi, M. Nagase, and K. Kurihara, J. Vac. Sci. Technol. B 16, 69 (1998).

${ }^{7}$ J. Kim, W. Chao, X. Liang, B. Griedel, D. Hilken, and D. Olynick, 53rd EIPBN Conference, Marco Island, FL, 2009 (unpublished), Paper No. 6B.5.

${ }^{8}$ G. M. Schmid, L. E. Carpenter, and J. A. Liddle, J. Vac. Sci. Technol. B 22, 3497 (2004).

${ }^{9}$ H. S. Lee, J. S. Lee, S. W. Nam, H. M. Kim, and K. B. Kim, J. Vac. Sci. Technol. B 27, 188 (2009).

${ }^{10}$ H. S. Lee, B. S. Kim, H. M. Kim, J. S. Wi, S. W. Nam, K. B. Jin, Y. Arai, and K. B. Kim, Adv. Mater. (Weinheim, Ger.) 19, 4189 (2007).

${ }^{11}$ S. Choi, M. J. Word, V. Kumar, and I. Adesida, J. Vac. Sci. Technol. B 26, 1654 (2008). 